\title{
Reversible methamphetamine-induced cardiomyopathy mimicking arrhythmogenic right ventricular cardiomyopathy with ventricular tachycardia
}

\author{
Aidan (Jia Sheng) Yu, MBBS, ${ }^{*}$ Matthew Rowe, MBBS, ${ }^{\dagger}$ Paul Martin, MBChB, ${ }^{*}$ \\ Arun Dahiya, MBBS* \\ From the *Royal Brisbane \& Women’s Hospital, Herston, Australia, and ${ }^{\dagger}$ Princess Alexandra Hospital, \\ Woolloongabba, Australia.
}

\section{Introduction}

Among numerous etiologies of ventricular arrhythmias, arrhythmogenic right ventricular cardiomyopathy (ARVC) and methamphetamine-induced cardiomyopathy (MAC) are 2 of the rarer causes of ventricular arrhythmias. ${ }^{1}$ ARVC is a disease that is characterized by fibro-fatty replacement of the myocardium. ${ }^{1}$ Although it was originally described as a right ventricular (RV) disease, left ventricular (LV) and biventricular involvement are also possible in patients with ARVC. ${ }^{1}$ On the other hand, MAC is a separate clinical entity that is rare and under-recognized. ${ }^{2}$

We report the first known case of reversible MAC mimicking ARVC with ventricular tachycardia (VT).

\section{Case report}

A 44-year-old white man presented with recurrent episodes of syncope on exertion. A nonsustained monomorphic VT, which was captured on his electrocardiogram (ECG) (Figure 1A), self-terminated but was associated with presyncope. His past medical history was significant for regular methamphetamine use, but he had no known medical conditions or family history of cardiac disorders. This patient did not have any cardiovascular risk factors. On average, he smoked $100 \mathrm{mg}$ of methamphetamine and ingested $100 \mathrm{mg}$ of liquid methamphetamine each week. He reported he last used intravenous methamphetamine a year ago. He also reported a 20-pack-year smoking history and drank 6 standard drinks per week. He denied using other illicit drugs.

A transthoracic echocardiogram was performed, which revealed impaired LV systolic function with an LV ejection fraction (LVEF) of 39\% (Simpson biplane). There was also

KEYWORDS Arrhythmogenic right ventricular cardiomyopathy; ARVD/C; Methamphetamine-induced cardiomyopathy; MAC; Ventricular tachycardia (Heart Rhythm Case Reports 2020;6:139-143)

Address reprint requests and correspondence: Dr Aidan (Jia Sheng) Yu, Royal Brisbane \& Women's Hospital, Butterfield St, Herston QLD 4029, Australia. E-mail address: aidanjsy@gmail.com.

\section{KEY TEACHING POINTS}

- Methamphetamine-induced cardiomyopathy (MAC) can mimic arrhythmogenic right ventricular cardiomyopathy (ARVC) with ventricular tachycardia. It is important to establish the correct diagnosis, as the disease process and long-term management differ significantly.

- The revised Task Force criteria are crucial in diagnosing ARVC but must be interpreted in the appropriate clinical context.

- Cardiac magnetic resonance imaging can be a useful tool in defining myocardial injury, predicting reversibility, and monitoring the progress of MAC.

mild concentric LV hypertrophy with global hypokinesis. Both left and right ventricles were borderline dilated. The right ventricular outflow tract (RVOT) parasternal longaxis RV dimension was $47 \mathrm{~mm}$, RVOT parasternal shortaxis RV dimension $49 \mathrm{~mm}$, and RV longitudinal myocardial velocity $7.6 \mathrm{~cm} / \mathrm{s}$. A coronary angiogram was performed and showed normal coronary arteries.

Further evaluation for ARVC included cardiac magnetic resonance imaging (MRI) with gadolinium enhancement. This showed multiple $\mathrm{RV}$ regional wall motion abnormalities with dyssynchronous contraction in the RVOT and apical RV free wall (Figure 2A and B, Supplementary Video 1). The sub-tricuspid RV free wall also appeared dyskinetic and there were areas of sub-segmental dyskinesis in the RVOT. The $\mathrm{RV}$ end-diastolic volume index was $87 \mathrm{~mL} / \mathrm{m}^{2}$ and $\mathrm{RV}$ ejection fraction (RVEF) was measured at $36 \%$. There was also global hypokinesis of the left ventricle with a measured LVEF of $35 \%$. There was no obvious gadolinium enhancement of both ventricles to suggest fibrotic or fat changes. 

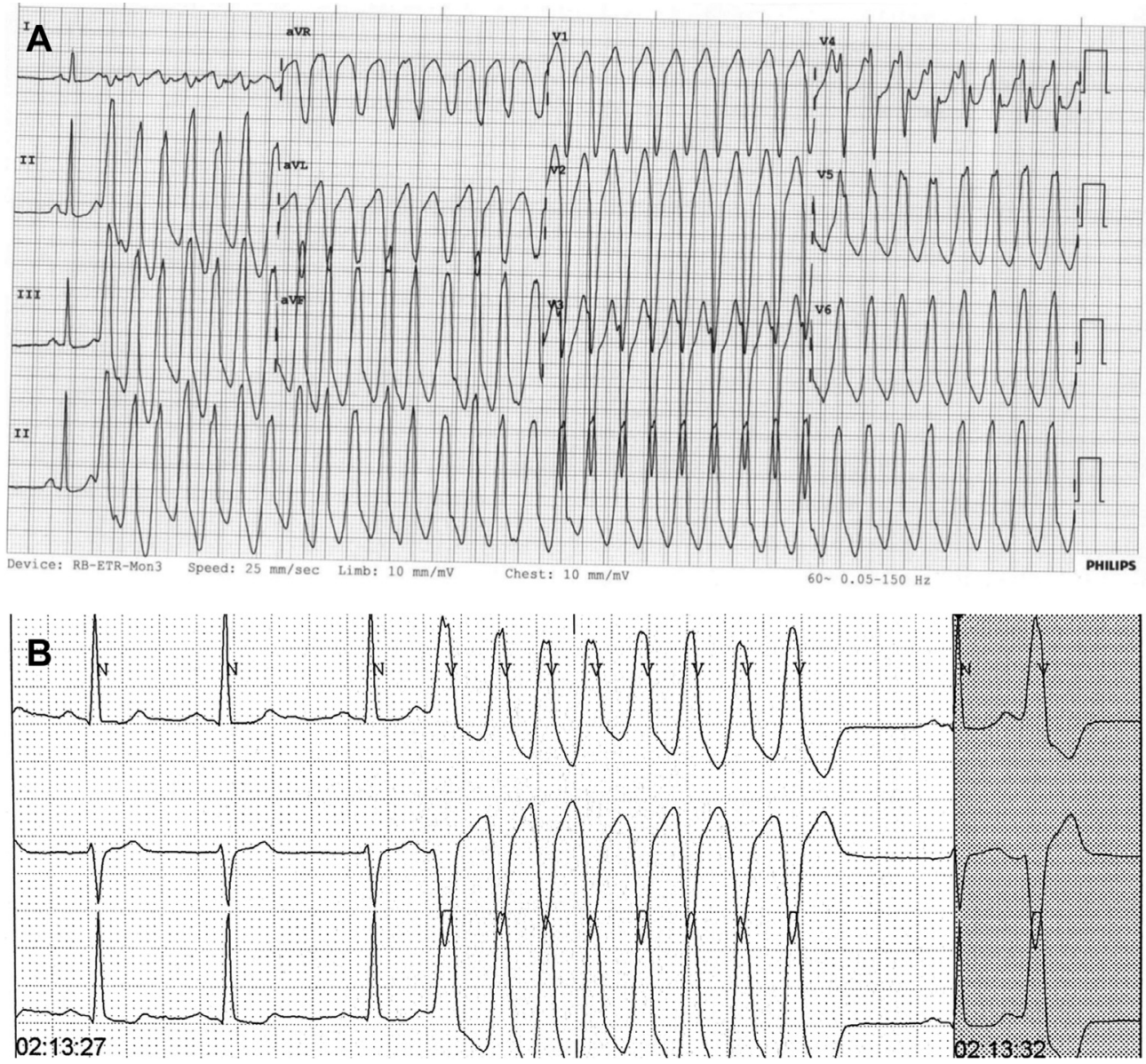

Figure 1 A: A 12-lead electrocardiogram demonstrating monomorphic ventricular tachycardia of right ventricular outflow tract morphology. B: Telemetry captured nonsustained monomorphic ventricular tachycardia of similar morphology following ingestion of methamphetamine on the ward.

A signal-averaged ECG (SAECG) was also conducted. This was positive, with a filtered QRS duration of $118 \mathrm{~ms}$ (normal $<114 \mathrm{~ms}$ ), root mean square voltage of terminal $40 \mathrm{~ms}$ of $38 \mu \mathrm{V}$ (normal $>20 \mu \mathrm{V}$ ), and duration of lowamplitude signal of $33 \mathrm{~ms}$ (normal $<38 \mathrm{~ms}$ ).

Under the Revised Task Force Criteria for ARVC, this patient met 1 major criterion (dyssynchronous RV contraction and RVEF $<40 \%$ ) and 2 minor criteria (nonsustained VT of left bundle branch block morphology with inferior axis, late potentials by SAECG), which meets the criteria for a definite diagnosis for ARVC. ${ }^{1}$

Electrophysiology study revealed no inducible sustained VT despite isoprenaline provocation. Voltage mapping of the right ventricle was performed using a 3-dimensional mapping system with bidirectional FlexAbility catheter (EnSite NavX; Abbott, Chicago, IL) and did not find any lowvoltage areas suggestive of fibrosis, which can be seen in ARVC.

Beta-blocker and angiotensin-converting enzyme inhibitors were initiated. The patient was monitored with telemetry on the ward and had been free of ventricular arrhythmia since admission. Nine days into his admission, his telemetry captured multiple episodes of monomorphic VT (Figure 1B) of similar morphology seen at presentation. On further questioning, he admitted to ingesting methamphetamine brought in by a friend. A decision was made to not insert an implantable cardioverterdefibrillator (ICD) in this admission given the clear temporal relationship of methamphetamine use and ventricular arrhythmia, freedom from arrhythmia on betablocker while abstaining from methamphetamine, and the risk of device infection given previous intravenous drug use. He was discharged on bisoprolol and perindopril. Drug and alcohol services were engaged to provide ongoing community detoxification therapy.

At the patient's 5-month follow-up appointment, he reported significantly reduced methamphetamine use. His repeat cardiac MRI showed resolution of previously seen RV segmental regional wall motion abnormalities and normalization of RV systolic function with an RVEF of 50\% (Figure 2C and D, Supplementary Video 2). In addition, there was no focal $\mathrm{LV}$ regional wall motion abnormality and LVEF had improved to 55\%. His outpatient 24-hour Holter monitor showed no VT. He demonstrated complete recovery of his cardiac function and the diagnosis of reversible cardiomyopathy owing to methamphetamine was made. We have continued follow-up for 18 months at the time of writing, and his course has been complicated by relapses in drug use and medication noncompliance, leading to further RVOT VT and syncope. 

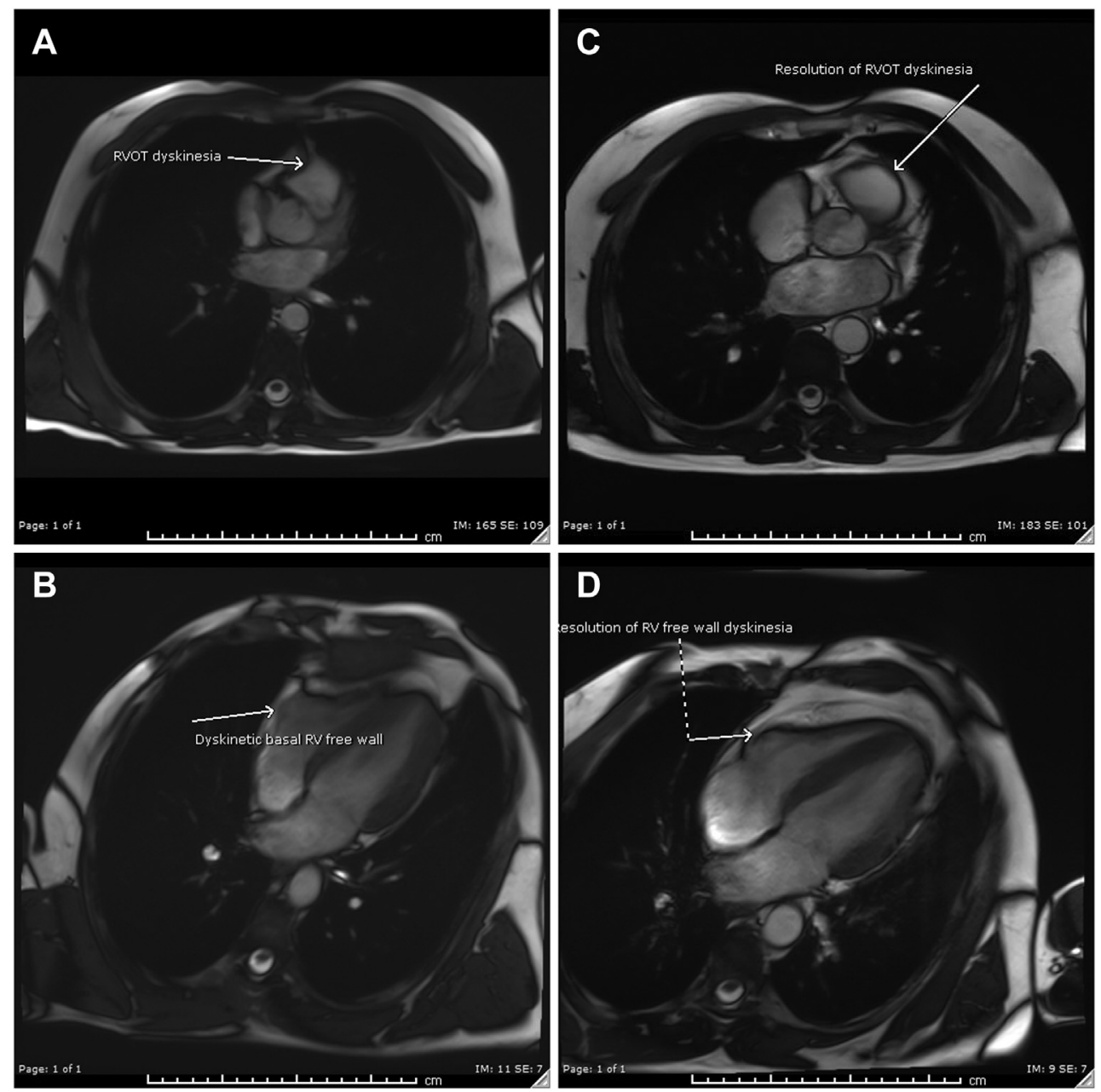

Figure 2 A: Cardiac magnetic resonance imaging demonstrating dyskinesia of right ventricular outflow tract (RVOT). B: Resolution of RVOT dyskinesia at 5month follow-up. C: Cardiac magnetic resonance imaging demonstrating dyskinetic basal right ventricular (RV) free wall. D: Resolution of RV free wall dyskinesia at 5-month follow-up.

\section{Discussion}

This case demonstrates the importance of establishing the correct underlying pathology of ventricular arrhythmia and the value of a thorough history including potential precipitants. Although our patient admitted to illicit drug use, many patients, for various reasons, may not. Methamphetamine use is common in Western countries such as Australia and, as such, should be directly inquired about.

Despite similar initial treatment approach to ventricular arrhythmias, the clinical course and long-term management for ARVC and MAC differ significantly. The placement of an ICD in a potentially reversible condition such as MAC would not only be unnecessary, but would also have exposed the patient to the risk of complications such as infection and inappropriate shocks.

Methamphetamine is a sympathomimetic agent that can result in stress-induced cardiomyopathy through inhibition of presynaptic catecholamine uptake and promotion of noradrenaline release. ${ }^{3}$ There are no pathognomonic imaging findings for MAC, but it can manifest as either acute LV impairment or chronic dysfunction or resemble Takotsubo syndrome. ${ }^{4}$ Past studies have shown that dilated cardiomyopathy is the most common type of cardiomyopathy associated with methamphetamine use. ${ }^{2,5}$ Dilatation of the ventricles was also evident in our case. In comparison to nonmethamphetamine users, patients with MAC are more prone to having increased LV end-diastolic volume, end-systolic volume, left atrial volume, and RV dimensions. ${ }^{5}$ In addition, methamphetamine users are known to have increased LV mass. ${ }^{5}$ This may be related to the increased sympathetic drive resulting in tachycardia and vasoconstriction that contribute to ventricular hypertrophy. ${ }^{5}$ Global LV systolic dysfunction with reduced LVEF is common in these patients.

Theories on the mechanism of MAC include coronary vasospasm and increased reactive oxygen species with resulting LV dilatation and systolic dysfunction. Methamphetamine use stimulates a hyperadrenergic state with resultant stressinduced cardiomyopathy through inhibition of presynaptic catecholamine uptake and promotion of noradrenaline release. ${ }^{4,5}$ Autopsy studies in MAC have revealed contraction band necrosis, normally found in catecholamine toxicity such as cocaine and pheochromocytoma-induced 
cardiomyopathy. ${ }^{4,5}$ In addition, methamphetamine also activates $\mathrm{Ca} /$ calmodulin-dependent protein kinase, which causes myocardial hypertrophy and fibrosis. ${ }^{5}$

A range of ECG abnormalities have been described in methamphetamine users. These abnormalities include prolonged QT interval, tachyarrhythmias, right axis deviation, LV hypertrophy, $\mathrm{P}$ pulmonale pattern, inferior $\mathrm{Q}$ waves, and lateral T-wave inversion. ${ }^{6}$ The most common ECG abnormality in this population is prolonged QTc, followed by nonspecific ST-T changes, sinus tachycardia, and sinus bradycardia. ${ }^{6}$ Notably, prolonged QTc increases the risk of arrhythmia, particularly polymorphic VT. In contrast, our patient's ECG demonstrated monomorphic VT following ingestion of methamphetamine. Monomorphic VT following methamphetamine use has been reported in 1 case report; however, this particular patient had entirely normal echocardiographic and cardiac MRI findings. ${ }^{7}$

A "reverse Takotsubo" (RT) pattern has also been described in methamphetamine users where there is hypokinesia or akinesia of the basal mid segments with apical preservation. The majority of these patients were female with a background of shorter duration of methamphetamine use. ${ }^{3}$ The RT pattern observed may be due to the distribution of adrenergic receptors. There is increased sympathetic innervation and fewer $\beta 2$ receptors in the basal region compared to the apical myocardium. Methamphetamine use increases not only concentrations of adrenaline, but also dopamine and noradrenaline. The $\beta 2$-adrenoceptors can suppress a high level of adrenaline but not noradrenaline. ${ }^{3}$ Therefore, the basal myocardium will be most affected, thus producing these wall motion abnormalities.

Reports of cardiac MRI findings on methamphetamine users are scarce. Findings that have been described are mid-wall delayed gadolinium enhancement, LV dilatation, and systolic dysfunction in methamphetamine users. ${ }^{3}$ In contrast, cardiac MRI findings in ARVC include regional RV wall motion abnormality (akinesis, dyskinesis, or dyssynchronous RV contraction), increased RV end-diastolic volume, depressed RVEF, intramyocardial fat, late gadolinium enhancement, and focal wall thinning. ${ }^{1,8}$ These abnormalities (RV dyssynchronous contraction and decreased RVEF) found in our patient resolved with reduced methamphetamine use.

Sporadic case reports have described reversibility of cardiomyopathy upon cessation of methamphetamine use. ${ }^{2,4,5}$ This may be owing to withdrawal of catecholamine surge and decreased adrenergic stress on the heart, similar to what is observed in stress-induced cardiomyopathy and pheochromocytoma-induced cardiomyopathy. In our case, despite poor compliance with beta-blockers and angiotensin-converting enzyme inhibitor therapy, this patient's cardiomyopathy resolved with reduction of methamphetamine use. The necessary dose and duration of methamphetamine usage required to develop cardiomyopathy is still unclear, as both acute and chronic forms of MAC have been reported. This may be owing to variations in individual response and tolerance to the drug. In theory, the absence of macroscopic fibrosis as shown by negative gadolinium enhancement is usually indicative of no permanent myocardial insult. This may be more indicative of a favorable prognosis. ${ }^{3,4}$ This correlated with our patient, whose cardiac MRI did not demonstrate gadolinium enhancement and who made an excellent recovery. In addition, echocardiographic features that favor early recovery include smaller left ventricular or left atrial size.

ARVC is a genetically determined disease characterized by fibro-fatty replacement of the myocardium. Although it predominantly affects the right ventricle, biventricular and left-dominant forms are now recognized. ${ }^{1}$ The disease is a result of desmosomal dysfunction when exposed to mechanical stress, culminating in myocyte separation and death. Inflammation ensues as the body attempts to repair the damaged myocytes, which produces fibro-fatty replacement of the myocardium. ${ }^{9}$ Both autosomal dominant and recessive patterns have been described, with the dominant pattern being more common. The genes involved include plakoglobin, desmoplakin, plakophilin-2, desmoglein-2, desmocollin-2, TMEM43, RyR2, and TGF-beta-3. ${ }^{9}$ The goal of treatment is to prevent sudden cardiac death and to slow disease progression. High-intensity and endurance sports are not recommended owing to the known risk of ventricular arrhythmias and disease progression. Beta-blockers and ICD play an important role in preventing sudden cardiac death. In addition, antiarrhythmic drugs such as sotalol can be used in patients with frequent ICD shocks, and rarely, cardiac transplantation is considered. ${ }^{10}$

Several disorders mimicking ARVC have been described. When these conditions involve the RV it is not uncommon for the task force criteria to be met. The well-known mimics are sarcoidosis and myocarditis, which may cause RV scarring and can be difficult to differentiate. ${ }^{11}$ Other conditions that could mimic RV pressure/volume overload on imaging include ostium secundum atrial septal defect, sinus venosus defect, severe tricuspid regurgitation, and pulmonary hypertension. ${ }^{11}$ However, the clinical history and associated findings of these other conditions are usually sufficient to separate them from ARVC.

SAECG is used to identify delayed and fragmented ventricular myocardial depolarization, which could provide the substrate for re-entrant arrhythmias. ${ }^{12}$ Kamath and colleagues ${ }^{12}$ found that using either 1 or 2 of the criteria (filtered QRS duration, root mean square voltage of terminal $40 \mathrm{~ms}$, low-amplitude signal) increased the sensitivity of diagnosing ARVC while maintaining high specificity. ${ }^{12}$ However, it is important to note that an abnormal SAECG is only a marker of RV cardiomyopathic process and not exclusive to ARVC. The abnormal SAECG in this case is interesting, as our patient did not have scar or slow conduction on MRI or electrophysiology study. We believe that the dyssynchrony in RV mechanical activation altered the QRS in much the same 
way LV dyssynchrony does in heart failure, resulting in a positive SAECG without true late potentials.

The original task force criteria proposed in 1994 to aid the diagnosis of ARVC were revised in 2010 to improve the sensitivity of diagnosing early disease and familial disease. ${ }^{1}$ This patient fulfilled 1 major and 2 minor criteria, which would have classified him as having a definite diagnosis of ARVC. However, it was evident that our patient's diagnosis was more consistent with MAC rather than ARVC given the history of methamphetamine use and normalization of cardiac function on follow-up.

\section{Conclusion}

To the best of our knowledge, this is the first reported case of reversible MAC mimicking ARVC with VT. Patients with a history of methamphetamine use who present with syncope and ventricular arrhythmias warrant a work-up for MAC. Establishing the correct diagnosis will aid long-term management and avoid unnecessary therapies. LV and RV dysfunction associated with methamphetamine use can be reversible with reduced drug use.

\section{Appendix}

\section{Supplementary data}

Supplementary data associated with this article can be found in the online version at https://doi.org/10.1016/j.hrcr.201 9.11.007.

\section{References}

1. Marcus FI, McKenna WJ, Sherrill D, et al. Diagnosis of arrhythmogenic right ventricular cardiomyopathy/dysplasia: proposed modification of the task force criteria. Circulation 2010;121:1533-1541.

2. Wijetunga M, Seto T, Lindsay J, Schatz I. Crystal methamphetamineassociated cardiomyopathy: tip of the iceberg? J Toxicol Clin Toxicol 2003;41:981-986.

3. Voskoboinik A, Ihle JF, Bloom JE, Kaye DM. Methamphetamine-associated cardiomyopathy: patterns and predictors of recovery. Intern Med J 2016; 46:723-727.

4. Lopez JE, Yeo K, Caputo G, Buonocore M, Schaefer S. Recovery of methamphetamine associated cardiomyopathy predicted by late gadolinium enhanced cardiovascular magnetic resonance. J Cardiovasc Magn Reson 2009; 11:46.

5. Ito H, Yeo KK, Wijetunga M, Seto TB, Tay K, Schatz IJ. A comparison of echocardiographic findings in young adults with cardiomyopathy: with and without a history of methamphetamine abuse. Clin Cardiol 2009;32:E18-E22.

6. Paratz ED, Zhao J, Sherwen AK, Scarlato RM, MacIsaac AI. Is an abnormal ECG just the tip of the ICE-berg? Examining the utility of electrocardiography in detecting methamphetamine-induced cardiac pathology. Heart Lung Circ 2017; 26:684-689.

7. Li J, Li J, Chen Y, et al. Methamphetamine use associated with monomorphic ventricular tachycardia. J Addict Med 2014;8:470-473.

8. Midiri M, Finazzo M, Brancato M, et al. Arrhythmogenic right ventricular dysplasia: MR features. Eur Radiol 1997;7:307.

9. Que D, Yang P, Song X, Liu L. Traditional vs. genetic pathogenesis of arrhythmogenic right ventricular cardiomyopathy. Europace 2015;17:1770-1776.

10. Corrado D, Wichter T, Link MS, et al. Treatment of arrhythmogenic right ventricular cardiomyopathy/dysplasia: an international task force consensus statement. Eur Heart J 2015;36:3227-3237.

11. Quarta G, Husain SI, Flett AS, et al. Arrhythmogenic right ventricular cardiomyopathy mimics: role of cardiovascular magnetic resonance. J Cardiovasc Magn Reson 2013;15:16.

12. Kamath GS, Zareba W, Delaney J, et al. Value of the signal-averaged electrocardiogram in arrhythmogenic right ventricular cardiomyopathy/dysplasia. Heart Rhythm 2011;8:256-262. 